\title{
SÍNTESIS DE SACARINATOCOBRE(I)
}

\author{
Mario Ceroni Galloso*a, Jorge R. Angulo Cornejo ${ }^{a}$, María N. Lino Pacheco ${ }^{a}$, \\ Claudia C. Villanueva Huerta ${ }^{a}$, Augusto G. Huiza Rojas ${ }^{\mathrm{a}}$
}

\begin{abstract}
RESUMEN
Se ha obtenido el compuesto de coordinación sacarinatocobre(I), $\left[\mathrm{Cu}_{2}\left(\mathrm{C}_{7} \mathrm{H}_{4} \mathrm{SO}_{3} \mathrm{~N}\right)_{2}\right]_{\mathrm{n}}$, por reacción de reducción de sulfato de cobre con ácido ascórbico y adición de sacarinato de sodio en medio acuoso. La caracterización se realizó por análisis elemental, espectroscopia fotoelectrónica de rayos $\mathrm{X}$, espectrofotometría infrarroja y voltametría cíclica.
\end{abstract}

Palabras clave: Cobre, sacarinato, XPS, IR.

\section{SYNTHESIS OF COPPER(I)- SACCHARINATE}

\begin{abstract}
The coordination compound saccharinatecopper(I), $\left[\mathrm{Cu}_{2}\left(\mathrm{C}_{7} \mathrm{H}_{4} \mathrm{SO}_{3} \mathrm{~N}\right)_{2}\right]_{\mathrm{n}}$, was obtained by reduction reaction of copper sulfate with ascorbic acid and addition of sodium saccharinate in aqueous medium. The characterization was performed by elemental analysis, $\mathrm{X}$ ray photoelectron spectroscopy, infrared spectrophotometry and cyclic voltammetry.
\end{abstract}

Key words: Copper, saccharinate, XPS, IR.

\section{INTRODUCCIÓN}

El sacarinato de sodio, desde 1885, es tal vez el edulcorante más usado en la industria de los productos alimenticios y en la formulación de algunos productos farmacéuticos; por ejemplo, en el endulzamiento de ciertos medicamentos, en la goma de mascar, enjuague bucal, pasta de dientes, aceite de hígado de bacalao y tabaco masticable. Por otra parte, desde 1955 se usa como aditivo en los baños de galvanizado de zinc para aumentar el brillo y la elasticidad de los revestimientos de níquel. ${ }^{1}$

La sacarina, mostrada en figura 1, es un compuesto heterociclo de importancia en la industria farmacéutica debido a que tiene en su estructura átomos de $\mathrm{C}, \mathrm{N}, \mathrm{O}$ y $\mathrm{S}$ relevantes en la

\footnotetext{
${ }^{a}$ Universidad Nacional Mayor de San Marcos, Facultad de Química e Ingeniería Química, Av. Germán Amézaga 375, Lima 1, Perú, mceronig@unmsm.edu.pe
} 
actividad de ciertas drogas ${ }^{2}$. Por ello, la sacarina y sus derivados orgánicos han mostrado muchas propiedades biológicas como inhibidores de: elastasa leucocitaria humana, catepsina grand proteasa, proteinasa ${ }^{3}$, aldehído deshidrogenasa ${ }^{4}$, agregación plaquetaria ${ }^{5}$, triptasa de los mastocitos humanos ${ }^{6}$, entre otros. Asimismo, se han estudiado propiedades analgésicas de los derivados de sacarina y como antídoto para intoxicación metálica ${ }^{8}$. Por otro lado, en la actualidad se estudia la reactividad de algunos compuestos metálicos de sacarina con el $\mathrm{ADN}^{9}$.<smiles>O=C1NS(=O)(=O)c2ccccc21</smiles>

Figura 1. Estructura de la sacarina.

La síntesis de nuevos derivados de la sacarina, tanto los orgánicos como los compuestos de coordinación, son de interés actual en la síntesis química. Debido a que la sacarina es un reactivo barato y muy versátil para la síntesis de nuevos derivados orgánicos heterociclos es posible realizar ataques nucleofílicos al carbono carbonílico ${ }^{10}$, la $\mathrm{N}$ y O- alquilación han producido muchos compuestos de gran valor para la química médica ${ }^{11}$ y recientemente se han obtenido nuevos compuestos producto de ciertas modificaciones en el anillo bencénico ${ }^{12}$.

La forma iónica de la sacarina (ion sacarinato), desde el punto de vista químico, es muy apreciada debido a su carácter polifuncional, por ello se convierte en un reactivo versátil capaz de coordinar diferentes metales de transición. ${ }^{13-14}$ Existen varios compuestos de sacarina con metales y en el caso de los compuestos con cobre todos los reportados son de cobre (II), pero muy pocos de cobre (I) ${ }^{15}$.

\section{PARTE EXPERIMENTAL}

Los equipos utilizados fueron los siguientes: Espectrofotómetro infrarrojo con transformada de Fourier Thermo Nicolet iS10, micro analizador elemental Leco Truspec Micro, UV-Vis Thermo Scientific-Helios, X-Ray photoelectron spectroscopy K-Alpha, resonancia magnética nuclear $\left({ }^{1} \mathrm{H}\right.$ 200MHz) Varian Gemini 200 y Bas Cyclic Voltammetry (CV) CV-1B-120 Serial 271 Bionalytical Bas Systems.

Los reactivos utilizados fueron de grado reactivo para síntesis y los solventes grado HPLC.

\section{Síntesis de sacarinatocobre(I)}

Se preparó una mezcla de ligando reductor, añadiendo $10 \mathrm{~mL}$ de solución acuosa de $0,07 \mathrm{~g}$ (0,40 mmol) de ácido ascórbico a $10 \mathrm{~mL}$ de solución acuosa de $0,19 \mathrm{~g}(0,79 \mathrm{mmol})$ de la sal de sacarinato de sodio. Esta mezcla se añade con agitación a una solución de $0,20 \mathrm{~g}(0,80$ mmol) de $\mathrm{CuSO}_{4} \cdot 5 \mathrm{H}_{2} \mathrm{O}$ en $10 \mathrm{~mL}$ de agua a temperatura ambiente. Luego de unos instantes 
se observó la aparición de una mezcla verde clara que fue variando su tonalidad a amarillo verdoso hasta adquirir una coloración amarillo perene con abundante precipitado. La mezcla se agitó durante 15 minutos más, se filtró, lavó con tres porciones de $10 \mathrm{~mL}$ de agua, el producto fue secado en estufa a $50{ }^{\circ} \mathrm{C}$ durante una hora y se obtuvo el producto de color amarillo. Rendimiento $90 \%$, p.f. mayor a $300{ }^{\circ} \mathrm{C}$.

Análisis elemental para $\mathrm{C}_{7} \mathrm{H}_{4} \mathrm{SO}_{3} \mathrm{NCu}$ calculado: $\mathrm{Cu} 25,90 \mathrm{C} 34,20, \mathrm{H} 1,60, \mathrm{~N} \mathrm{5,70} \mathrm{y} \mathrm{S}$ 13,10\%. Encontrado: Cu 25,80 C 34,20, H 1,76, N 5,46 y S 13,00\%.

FT-IR $\left(\mathrm{cm}^{-1}\right): 3080 \mathrm{w}\left(\mathrm{CH}_{\text {arom }}\right), 1609 \mathrm{~s}(\mathrm{C}=\mathrm{O}), 1578 \mathrm{~s}\left(\mathrm{C}=\mathrm{C}_{\text {arom }}\right), 1458 \mathrm{~m}\left(\mathrm{C}=\mathrm{C}_{\text {arom }}\right), 1296 \mathrm{~s}$ $\left(\mathrm{SO}_{2}\right), 1262 \mathrm{~m}(\mathrm{C}=\mathrm{O}), 1146 \mathrm{vs}\left(\mathrm{SO}_{2}\right), 1115 \mathrm{~s}(\mathrm{CH}), 1049 \mathrm{~m}(\mathrm{CH}), 945 \mathrm{~s}(\mathrm{CH}), 752 \mathrm{~s}(\mathrm{CH})$.

UV-Vis $\lambda_{\text {max }}(\mathrm{DMF}) / \mathrm{nm}: 290$ y 789.

XPS (eV): N 1s 399, 15 eV; O 1s 532,7 eV y 531,49 eV. S 2p 168,4 eV; C 1s 285,08, 288,21 y $286,01 \mathrm{eV} ; \mathrm{Cu} 2 \mathrm{p} 3 / 2$ 932,76 eV.

${ }^{1} \mathrm{H} \mathrm{RMN}\left(200 \mathrm{MHz}, \mathrm{CD}_{2} \mathrm{Cl}_{2}, \mathrm{Me}_{4} \mathrm{Si}\right) \delta: 7,87(1 \mathrm{H}, \mathrm{d}), 7,6-7,8(3 \mathrm{H})$.

\section{RESULTADOS Y DISCUSIÓN}

El compuesto obtenido corresponde a un sólido de color amarillo intenso que se obtuvo gracias a una reacción template, en la que se forma en primer lugar el complejo de sacariantocobre(II) de color verde y luego se produce la reducción de cobre (II) a cobre (I), haciendo que la reacción sea específica. Normalmente los complejos de cobre (I) son de color amarillo y tienden a formar polímeros insolubles. La razón de este actuar se basa en la inestabilidad que presenta la especie $\mathrm{Cu}^{\mathrm{I}}$ de asociarse a iones libres que se encuentren en el medio y que pudieran dar productos no deseados, justamente es en este punto donde actúa el ion sacarinato.

Respecto a la solubilidad el compuesto sintetizado es térmicamente inestable en todos los solventes tratados y que se disuelve en frío solo con solventes muy polares y coordinantes como DMF, DMSO y piridina.

El análisis elemental encontrado se aproxima al análisis elemental calculado, con lo cual se obtiene la fórmula empírica $\mathrm{C}_{7} \mathrm{H}_{4} \mathrm{SO}_{3} \mathrm{NCu}$. Se sabe, en general, que la estequiometria da pocos indicios sobre la estructura de los compuestos: por ello, este compuesto podría ser mononuclear, binuclear o polinuclear.

La naturaleza de coordinación del cobre (I) es usualmente dos y tres ${ }^{16}$, sumado al análisis IR del nuevo complejo que presenta una sola señal del enlace $\mathrm{C}=\mathrm{O}$ desplazado hacia frecuencias muy bajas $\left(1609 \mathrm{~cm}^{-1}\right)$ lo cual es una evidencia de enlace oxígeno del grupo amido $-\mathrm{NC}=\mathrm{O}$ unido a cobre. Por ello, posiblemente el compuesto tenga una estructura de naturaleza no 
monomérica constituida por unidades fundamentales del tipo $\left[\mathrm{Cu}\left(\mathrm{C}_{7} \mathrm{H}_{4} \mathrm{SO}_{3} \mathrm{~N}\right)\right]$, con enlaces $\mathrm{Cu}-\mathrm{N}_{\mathrm{sac}}$ y además el $\mathrm{C}=\mathrm{O}$ del ion sacarinato interactúa también con el $\mathrm{Cu}^{\mathrm{I}}$.

En general, los compuesto de cobre (I) $\left(\mathrm{d}^{10}\right)$ son incoloros, pero pueden ser coloreados a causa del ligando o la aparición de bandas de transferencia de cargas. Como el sacarinato es incoloro, el compuesto amarillo verdoso obtenido debe su color a las transferencias de cargas de átomos dadores como el $\mathrm{C}=\mathrm{O}$ de la sacarina al cobre (I). Por ello, el segundo pico intenso $\mathrm{UV}$-Vis que aparece a $789 \mathrm{~nm}$ está ligado a este fenómeno.

El estudio del espectro ${ }^{1} \mathrm{H}$ RMN indica la presencia del anión sacarinato, se observan las señales de los protones aromáticos. Los protones del tipo (2), (3) y (4) están contenidos en una sola señal ancha y muy intensa con un desplazamiento químico entre 7,6 y 7,8 ppm, mientras que el protón (1) se muestra como un doblete a 7,87 ppm.

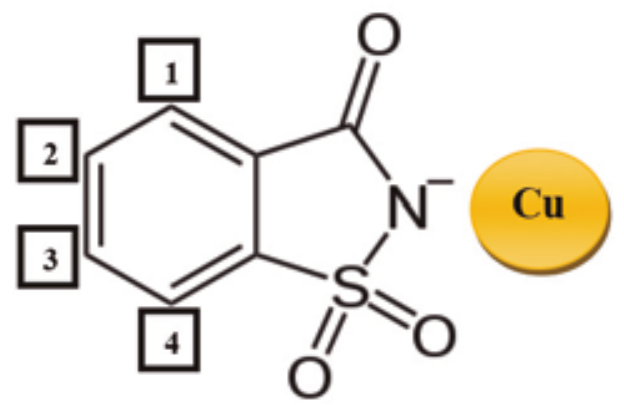

Figura 2. Protones del grupo sacarinato del compuesto obtenido.

En el espectro RMN ${ }^{13} \mathrm{C}$, si bien las señales son anchas y por ello no se observan los 7 carbonos, se puede apreciar la del carbonilo de la sacarina coordinada al cobre(I) a 166 ppm. La señal del $\mathrm{CO}$ del dímero $\left[\mathrm{Cu}(\mathrm{sac})\left(\mathrm{PPh}_{3}\right)\right]_{2}{ }^{15}$ aparece a $168 \mathrm{ppm}$ y en el caso de la sacarina libre aparece a $161 \mathrm{ppm}$. Este dato corrobora la gran interacción de ese grupo carbonilo con el cobre, tal como se observó también en el FTIR. Por ello, ambos datos indican una coordinación del grupo carbonilo al cobre(I).

Se ha realizado voltamperogramas cíclicos (CVs) de 1000 ppm del complejo obtenido en acetonitrilo $6,0 \%$ en el rango de potencial de $-1,5$ a 1,5 $\mathrm{V}$ y a diferentes velocidades de barrido $(75,100$ y $300 \mathrm{mV} / \mathrm{s})$. Se ha determinado también que todos los voltamperogramas realizados no muestran la oxidación ni reducción íntegra del ligando sacarinato ni mucho menos la descomposición del complejo para el rango del potencial de -1,5 a 1,5 V. Igualmente, se puede observar que la relación de picos de corriente $\left(\mathrm{I}_{\mathrm{pc}} / \mathrm{I}_{\mathrm{pa}}\right)$ tiene una ligera aproximación a 1 y los valores de $\Delta \mathrm{E}\left(=\left|\mathrm{E}_{\mathrm{pa}}-\mathrm{E}_{\mathrm{pc}}\right|\right)$ son muy altos en relación al sistema reversible $(60 \mathrm{mV})$. Esto sugiere, por consiguiente, que el fenómeno está relacionado al salto de solo un electrón cuya transferencia no está en equilibrio y es ligeramente rápida. Además, se puede observar que en cada uno de los casos los potenciales de pico anódico y catódico son independientes 
de la velocidad de barrido. Por lo tanto, con todos los datos obtenidos se puede considerar que el compuesto exhibe solamente electroactividad en el centro metálico pero el sistema redox $\mathrm{Cu}(\mathrm{II}) / \mathrm{Cu}(\mathrm{I})$ se orienta mejor al comportamiento cuasi-reversible.

Por otro lado, para el espectro XPS de $\mathrm{Cu} 2 \mathrm{p}^{3 / 2}$ (figura 3) confirma que la señal a 932,76 eV corresponde únicamente a la presencia de CuI coordinado.17 Las señales XPS para el O1S confirman la formación de un enlace $\mathrm{Cu}-\mathrm{O}(532,7 \mathrm{eV})^{17}$; a 531,80 eV se observa la señal de doble intensidad que la señal del anterior es asignada al $\mathrm{SO}_{2}$. El XPS para el N1s indica la presencia de enlace $\mathrm{N}-\mathrm{Cu}$ a $399,15 \mathrm{eV}$.
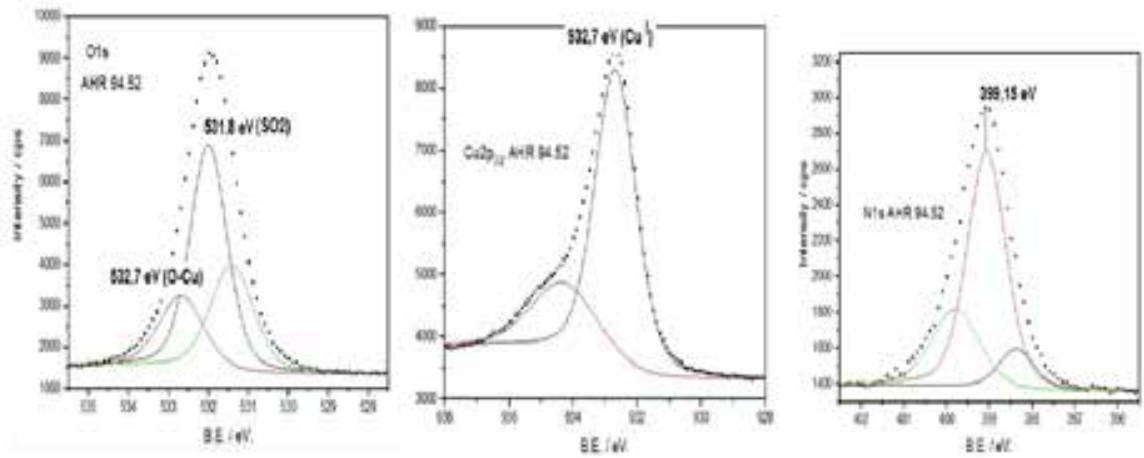

Figura 3. Señales XPS Cu2 $\mathrm{p}_{3 / 2}, \mathrm{~N} 1 \mathrm{~S}$ y O1S del complejo $\left[\mathrm{Cu}_{2}\left(\mathrm{C}_{7} \mathrm{H}_{4} \mathrm{SO}_{3} \mathrm{~N}\right)_{2}\right]_{\mathrm{n}}$

Por el análisis del IR y XPS se puede afirmar que el compuesto obtenido tiene el cobre en estado de oxidación +1 y está unido a través del nitrógeno y oxígeno del grupo $\mathrm{C}=\mathrm{O}$ del sacarinato, formando un puente. Se trataría, en realidad, de una estructura básica dimérica mostrada en la figura 4.

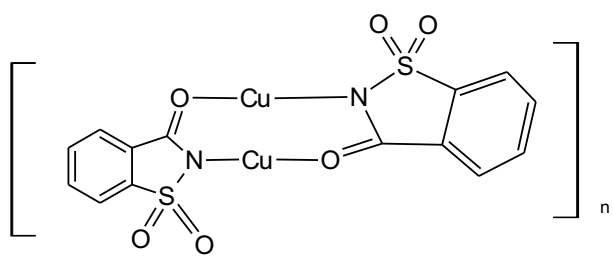

Figura 4. Estructura dimérica del compuesto $\left[\mathrm{Cu}_{2}\left(\mathrm{C}_{7} \mathrm{H}_{4} \mathrm{SO}_{3} \mathrm{~N}\right)_{2}\right]_{\mathrm{n}}$

La estructura dimérica de cobre(I) donde hay enlaces $\mathrm{Cu}-\mathrm{O}=\mathrm{C}$ y $\mathrm{Cu}-\mathrm{N}$, ambos con dos sacarinas ya fue reportada en el compuesto $\left[\mathrm{Cu}(\mathrm{sac})\left(\mathrm{PPh}_{3}\right)\right]_{2}{ }^{15}$, donde además cada cobre coordina con una trifenilfosfina. Asimismo, este tipo de enlace se encuentra en el compuesto de coordinación de cobre(II) en $\left[\mathrm{Cu}_{2}\left(\mathrm{C}_{6} \mathrm{H}_{4} \mathrm{COSO}_{2} \mathrm{~N}\right)_{4}\left(\mathrm{C}_{3} \mathrm{H}_{4} \mathrm{~N}_{2}\right)_{4}\right]^{18}$. 


\section{CONCLUSIONES}

Se ha obtenido un nuevo compuesto de coordinación entre el ion sacarinato y el cobre(I) mediante una reacción de reducción template, el cual genera el complejo de fórmula $\left[\mathrm{Cu}_{2}\left(\mathrm{C}_{7} \mathrm{H}_{4} \mathrm{SO}_{3} \mathrm{~N}\right)_{2}\right]_{\mathrm{n}}$ con una estructura probablemente dimérica donde el sacarinato actúa como un ligando puente a través del $\mathrm{N}$ y del $\mathrm{C}=\mathrm{O}$.

Los datos de la voltametría cíclica indican que el compuesto exhibe solamente electroactividad en el centro metálico pero el sistema redox $\mathrm{Cu}(\mathrm{II}) / \mathrm{Cu}(\mathrm{I})$ se orienta mejor al comportamiento cuasi-reversible.

\section{AGRADECIMIENTO}

Los autores agradecen a Innóvate Perú por el contrato No 364-PNICP-PIBA-2014. Asimismo, al Dr. Lothar Hennig de la Universidad de Leipzig, Alemania, por los espectros RMN.

\section{BIBLIOGRAFÍA}

1. Roth K, Lück E. Die Saccharin-Saga. Chem Unserer Zeit. 2011; 45:406-423.

2. Yeung K,Meanwell NA,Li Y,Gao Q.A facile construction of 4-hydroxymethylbenzisothiazolone-1,1-dioxide.Tetrahedron Lett. 1998; 39:1483-1486. https://doi.org/10.1016/S0040-4039(98)00011-2.

3. Groutas WC, Kuang R, Ruan S, Epp JB, Venkataraman R, Truong TM. Potent and specific inhibition of human leukocyte elastase,cathepsin $\mathrm{G}$ and proteinase 3 by sulfone derivatives employing the 1,2,5-thiadiazolidin-3-one 1,1 dioxide scaffold. Bioorg Med Chem.1998;6: 71-661.

4. Nagasawa HT, Kawle SP, Elberling JA, DeMaster EG, Fukuto JM. Prodrugs of nitroxyl as potential aldehyde dehydrogenase inhibitors vis-a-vis vascular smooth muscle relaxants. J Med Chem.1995;38: 71-1865.

5. Sunkel CE, Fau de Casa-Juana M, Cillero FJ, Priego JG, Ortega MP. Synthesis, platelet aggregation inhibitory activity,and in vivo antithrombotic activity of new 1,4-dihydropyridines. J Med Chem.1988;31: 90-1886.

6. Combrink KD, Gülgeze HB, Meanwell NA, Pearce BC, Zulan P, Bisacchi GS, et al. 1,2-Benzisothiazol-3-one 1,1-dioxide inhibitors of human mast cell tryptase. J Med Chem.1998;41:60-4854.

7. González G, Lyndon C, Sunkel C. Hepatic Kinetics of SCP-1(N-[alpha-(1,2benzisothiazol-3(2H)-ona-1,1-dioxide-2-yl)-acetyl]-p-aminophenol) compared with acetaminophen in isolated rat liver. Eur J Pharm Biopharm.1998;46:7-293.

8. Malik KMA, Haider SZ, Hossain MA, Hursthouse MB. Dipotassium Sodium Trisaccharinate Monohydrate. Acta Cryst.1984;40:1696-1698.

9. Kumar P, Dasari S, Patra AK. Ruthenium (II) complexes of saccharin with dipyridoquinoxaline and dipyridophenazine: Structures,biological interactions and 
photoinduced DNA damage activity. Eur J Med Chem.2017;136:52-62.

10. Maiti S, Rambabu D, Prasad ASG, Rao VG, Rao MVB. Synthesis and Utilization of Saccharin Derivatives. JOAC.2012;1:467-477.

11. Jakopin ž, Dolenc MS. Advances in the Chemistry of Saccharins: From Synthetic Novelties Towards Biologically Active Compounds. Curr Med Chem. 2010:17:651671.

12. Rankin GM, Poulsen S. Synthesis of Novel Saccharin Derivatives. MDPI. 2017; 22:516. DOI: $10.3390 /$ molecules22040516.

13. Baran EJ, Yilmaz VT. Metal complexes of saccharin. Coord Chem Rev.2006; 250:19801999.

14. Jovanovski G. Metal Saccharinates and Their Complexes with N-donor Ligands. CCACAA. 2000; 73:843-868.

15. Falvello LR, Gomez J, Pacual I, Tomás M, Urriolabeiltia, Schultz AJ. Saccharinate as a versatile Polyfunctional Ligand. Four Distinct coordinations Modes. Misdirected Valence, and a Dominant Aggregate Structure from a single Reaction System. Inorg Chem. 2001; 40: 4455-4463.

16. Emeléus HJ, Sharpe AG.Advances in Inorganic Chemistry and Radiochemistry. 17th Ed. Cambridge: Editorial Elsevier; 1975.

17. Lino-Pacheco MN. Síntesis y caracterización de ligandos quelantes y sus complejos metálicos: estructura molecular del complejo bis[N-benzoil-O-metil-N'-(p-nitrofenil)isoureato]cobre(II) [tesis de maestría]. Lima: Universidad Nacional Mayor de San Marcos, Facultad de Química e Ingeniería Química; 2000.

18. Liu SH, Huang JL. Structure of a Copper Complex Containig Saccharin and Imidazole: $\left[\mathrm{Cu}_{2}\left(\mathrm{C}_{6} \mathrm{H}_{4} \mathrm{COSO}_{2} \mathrm{~N}\right)_{4}\left(\mathrm{C}_{3} \mathrm{H}_{4} \mathrm{~N}_{2}\right)_{4}\right]$. Acta Cryst. 1991; C47:41-43. 